thus identified as successive divided differences, and the formula (3) itself is Newton's formula.

We can go on to prove that divided differences are symmetrical in their arguments: for example $u(a, b, c)$ is seen from our work to be the coefficient of the highest power of $x$ in the polynomial determined by $u_{a}, u_{t}, u_{c}$; and this is the same whatever the order taken. Also the $n^{\text {th }}$ divided difference derived from $n+1$ values of a polynomial of the $n^{\text {th }}$ degree is constant and independent of its arguments; for it is the coefficient of the highest power of $x$ in the polynomial, which is fixed.

\title{
A Note on Mathematical Expectation
}

\author{
By A. C. Aitken.
}

If there are $n$ coins in a bag, of any individual values but of total value $P$, and if $m$ are drawn at random, without replacement, it may be proved that the mathematical expectation, or probable value, of the draw is $m P / n$. It must be well known, though I cannot find any reference in the texts, that the same result holds if the coins are replaced after each drawing, or indeed if replacements after certain specified drawings, but not after others, are permitted.

It may therefore be worth while to prove the following result:

If there are $n$ coins, of any individual values, and of total value $P$, and if $m$ are to be drawn by any rule that does not mention individual coins (e.g. with replacements, or without replacements, or with replacements at certain agreed stages), then the probable value of the draw is $m P / n$.

Proof. The $n$ coins, regarded as possessing distinct identity (though there may be groups of the same denomination), may be denoted by $k_{1}, k_{2}, \ldots, k_{n}$.

Let the number of different possible drawings of $m$ of these, under the rules agreed upon, and order being taken into account, be $N$, and let one such order be

$$
k_{\alpha}, k_{\beta}, \ldots, k_{\mu},
$$

where $a, \beta, \ldots, \mu$ are $m$ numbers chosen from $1,2, \ldots, n$, in which 
$x v i$

of course there may be repetitions if replacements are allowed. The part of the probable value due to this drawing is

$$
\frac{1}{N}\left(k_{\alpha}+k_{\beta}+\ldots+k_{\mu}\right)
$$

and the total probable value is

$$
\frac{1}{N} \Sigma\left(k_{\alpha}+k_{\beta}+\ldots+k_{\mu}\right)
$$

where $\Sigma$ denotes summation of $N$ sets of $m$ terms each, in all $m N$ terms.

Now, since no coin is singled out for special favour, $\alpha$ will equally often be $1,2, \ldots$, or $n$; and the same is true of $\beta, \gamma, \ldots$, and $\mu$. Hence in the sum above the expression $k_{1}+k_{2}+\ldots+k_{n}$ must occur a whole number of times, and this whole number must be $m N / n$. Thus finally, since the value of all the coins is $P$, the probable value in question is

$$
\frac{1}{N} \cdot \frac{m N}{n} \cdot P=\frac{m P}{n} .
$$

The invariant property of mathematical expectation is thus brought out. (For a rather similar result see Chrystal's Algebra, Part II, pp. 594-5.)

The case where the number of replacements allowed is not limited leads to an identity in combinatory analysis which is by no means obvious, namely

$$
\sum_{r=1}^{m} \frac{m !}{m_{1} ! m_{2} ! \ldots m_{r} !}\left(m_{1} a_{1}+m_{2} a_{2}+\ldots+m_{r} a_{r}\right)=m n^{m-1}\left(a_{1}+a_{2}+\ldots+a_{1}\right.
$$

where $m_{1}+m_{2}+\ldots+m_{r}=m$, and $\Sigma$ includes all $r$-part compositions (i.e. partitions in which order of parts is relevant) of $m$, associated with all $r$-ary combinations of $1,2, \ldots, n$.

\section{A Simple Method of Finding Sums of Powers of the Natural Numbers}

By I. M. H. Etherington.

Let $1^{a}+2^{a}+3^{a}+\ldots+n^{a}$ be denoted by $S_{\alpha}$. It is well known that $S_{\alpha}$ can be expressed as a polynomial in $n$ of degree $(\alpha+1)$. The expressions for $S_{1}, S_{2}, S_{3} \ldots$ can be found in succession by elementary methods, which also give numerous relations such as $S_{3}=S_{1}^{2}$, 